\title{
Systems biology of vaccination for seasonal influenza in humans
}

\author{
Helder I Nakaya ${ }^{1,2}$, Jens Wrammert ${ }^{1,3}$, Eva K Lee ${ }^{4}$, Luigi Racioppi ${ }^{5,6}$, Stephanie Marie-Kunze ${ }^{1,2}$, W Nicholas Haining ${ }^{7}$, \\ Anthony R Means ${ }^{6}$, Sudhir P Kasturi ${ }^{1,2}$, Nooruddin Khan ${ }^{1,2}$, Gui-Mei Li ${ }^{1,3}$, Megan McCausland ${ }^{1,3}$, Vibhu Kanchan ${ }^{1,3}$, \\ Kenneth E Kokko ${ }^{8}$, Shuzhao Li ${ }^{1,2}$, Rivka Elbein ${ }^{9}$, Aneesh K Mehta ${ }^{9}$, Alan Aderem ${ }^{10}$, Kanta Subbarao ${ }^{11}$, Rafi Ahmed ${ }^{1,3}$ \\ \& Bali Pulendran ${ }^{1,2,12}$
}

\begin{abstract}
Here we have used a systems biology approach to study innate and adaptive responses to vaccination against influenza in humans during three consecutive influenza seasons. We studied healthy adults vaccinated with trivalent inactivated influenza vaccine (TIV) or live attenuated influenza vaccine (LAIV). TIV induced higher antibody titers and more plasmablasts than LAIV did. In subjects vaccinated with TIV, early molecular signatures correlated with and could be used to accurately predict later antibody titers in two independent trials. Notably, expression of the kinase CaMKIV at day 3 was inversely correlated with later antibody titers. Vaccination of CaMKIV-deficient mice with TIV induced enhanced antigen-specific antibody titers, which demonstrated an unappreciated role for CaMKIV in the regulation of antibody responses. Thus, systems approaches can be used to predict immunogenicity and provide new mechanistic insights about vaccines.
\end{abstract}

Annual vaccination is one of the most effective methods for preventing influenza ${ }^{1}$. At present, two types of vaccines for seasonal influenza are licensed for use in the USA: trivalent inactivated influenza vaccine (TIV), given by intramuscular injection; and live attenuated influenza vaccine (LAIV), administered intranasally. These vaccines contain three strains of influenza viruses that are usually changed annually on the basis of the results of global influenza surveillance data ${ }^{2}$. The efficacy of a vaccine against influenza, therefore, depends on the match of antigenicity between the vaccine and circulating influenza strains ${ }^{3}$. Additionally, other factors such as the age and immunocompetence of vaccinees, as well as preexisting amounts of antibody derived from prior infection or vaccination, contribute to mechanisms that mediate the efficacy of vaccines against influenza ${ }^{1,2,4}$.

Systems vaccinology has emerged as an interdisciplinary field that combines systems-wide measurements plus network and predictive modeling applied to vaccinology ${ }^{5}$. A systems biology approach has been used to identify early gene signatures that correlate with and can be used to predict later immune responses in humans vaccinated with the live attenuated vaccine YF-17D against yellow fever ${ }^{6,7}$. YF-17D is one of the most successful vaccines ever developed ${ }^{8,9}$; it stimulates polyvalent innate immune responses ${ }^{10}$ and adaptive immune responses ${ }^{11}$ that can persist for decades after vaccination ${ }^{11}$. Although systems biology approaches have been used to predict the immunogenicity of YF-17D 6,7 , which is a live replicating virus, the extent to which such approaches can be applied to the prediction of the immunogenicity of inactivated vaccines is unknown. Furthermore, it remains unclear whether systems approaches can be used to predict the immunogenicity of recall responses. In the case of influenza, the immune response to vaccination is greatly enhanced by the past history of the vaccine recipient, both by prior infections and vaccinations. Notably, whether such approaches can provide insight into the immunological mechanisms of action of vaccines and help with the discovery of new correlates of protective immunity is untested. To address these issues, we did a series of clinical studies during the annual influenza seasons in 2007, 2008 and 2009, in which we vaccinated healthy young adults with TIV. Our goal was to undertake a detailed characterization of the innate and adaptive responses to vaccination with TIV to identify putative early signatures that correlated with or could be used to predict later immunogenicity and to obtain new insight into the mechanisms that underlie immunogenicity.

The results of our studies demonstrate that systems biology approaches can indeed be used to predict the immunogenicity of an inactivated vaccine such as TIV with up to $90 \%$ accuracy. Notably, the expression at day 3 of one of the genes in the predictive

\footnotetext{
${ }^{1}$ Emory Vaccine Center, Emory University, Atlanta, Georgia, USA. ${ }^{2}$ Yerkes National Primate Research Center, Emory University, Atlanta, Georgia, USA. ${ }^{3}$ Department of Microbiology and Immunology, Emory University, Atlanta, Georgia, USA. ${ }^{4}$ Center for Operations Research in Medicine \& Healthcare, School of Industrial \& Systems Engineering, Georgia Institute of Technology, Atlanta, Georgia, USA. ${ }^{5}$ Department of Pharmacology and Cancer Biology, Duke University, Durham, North Carolina, USA. ${ }^{6}$ Department of Cellular and Molecular Biology and Pathology, University of Naples Federico II, Naples, Italy. ${ }^{7}$ Dana-Farber Cancer Institute, Boston, Massachusetts, USA. ${ }^{8}$ Department of Medicine, Division of Nephrology, Emory University School of Medicine, Atlanta, Georgia, USA. ${ }^{9}$ Division of Infectious Diseases, Department of Medicine, School of Medicine, Emory University, Atlanta, Georgia, USA. ${ }^{10}$ Institute for Systems Biology, Seattle, Washington, USA. ${ }^{11}$ Laboratory of Infectious Diseases, National Institute for Allergy and Infectious Diseases, National Institutes of Health, Bethesda, Maryland, USA. ${ }^{2}$ Department of Pathology, Emory University School of Medicine, Atlanta, Georgia, USA. Correspondence should be addressed to B.P. (bpulend@emory.edu).
} 
signature, encoding the kinase CaMKIV, was inversely correlated with plasma hemagglutination-inhibition (HAI) antibody titers at day 28. Vaccination of CaMKIV-deficient $\left(\mathrm{Camk}^{-/-}\right)$mice with TIV induced enhanced antigen-specific antibody titers, which demonstrated an unappreciated role for CaMKIV in the regulation of antibody responses. Together our results demonstrate the utility of systems biology not only in the prediction of vaccine immunogenicity but also in offering new insight into the molecular mechanism of influenza vaccines.

\section{RESULTS}

\section{Antibody responses induced by TIV and LAIV}

We evaluated the antibody responses of 56 healthy young adults vaccinated with either LAIV $(n=28)$ or TIV $(n=28)$ during the 2008 influenza season. We determined HAI titers for each of the three influenza strains in LAIV and TIV in the plasma of vaccinees at baseline (day 0 ) and at $28 \mathrm{~d}$ after vaccination. We calculated the magnitude of antibody responses to the vaccine (HAI response) as the maximum difference between the HAI titer at day 28 and the baseline titer (day 0 ) for any of the three influenza strains contained in the vaccine (Fig. 1a). The mean HAI response of subjects vaccinated with TIV was sixfold higher than that of those vaccinated with LAIV (Fig. 1a), consistent with many published reports ${ }^{1,12,13}$. Furthermore, among the subjects vaccinated with TIV, there was considerable variation in the magnitude of the HAI response ( $>100$-fold; Fig. 1a). According to the US Food and Drug Administration Guidance for Industry document for this field ${ }^{14}$, seroconversion can be defined by an HAI titer of 1:40 or more and a minimum fourfold increase in antibody titer after vaccination. Thus, we operationally classified the vaccinees as 'low HAI responders' or 'high HAI responders' based on whether or not a fourfold increase occurred after vaccination (Fig. 1a). Most of the subjects vaccinated with TIV (22 of 28) were classified as high HAI responders; only six
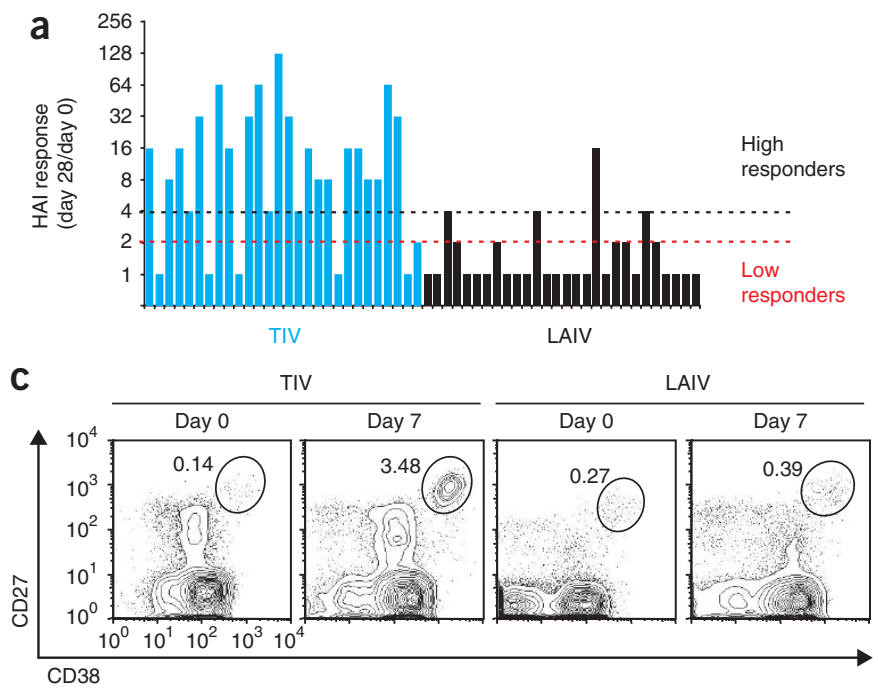

were classified as low HAI responders. In contrast, most subjects vaccinated with LAIV (24 of 28) were classified as low HAI responders and only four were classified as high HAI responders (Fig. 1a).

Antibodies are produced by antibody-secreting B cells in the blood (plasmablasts) or bone marrow and secondary lymphoid organs (fully differentiated plasma cells). High frequencies of antigen-specific plasmablasts in the blood within a few days of vaccination, reaching a peak at day 7 , have been documented ${ }^{15}$. To determine whether the early plasmablast response to influenza vaccination correlated with the later HAI response, we assessed the frequency of influenza-specific plasmablasts at baseline and $7 \mathrm{~d}$ after vaccination (Fig. 1b,c). As reported before ${ }^{15}$, we observed rapid clonal expansion of influenzaspecific plasmablasts $7 \mathrm{~d}$ after vaccination with TIV, as measured by enzyme-linked immunospot (ELISPOT) assay (Fig. 1b) and by flow cytometry (Fig. 1c). We further found that the population expansion of circulating plasmablasts secreting immunoglobulin $\mathrm{G}$ (IgG) was also greater in subjects vaccinated with TIV than in those vaccinated with LAIV (Fig. 1b,c). We obtained similar results for IgA-secreting plasmablasts at day 7 after vaccination (Supplementary Fig. 1a), and a very good correlation was evident between the frequency of plasmablasts as measured by ELISPOT and their frequency as measured by flow cytometry (Fig. 1d and Supplementary Fig. 1b).

As we detected a very low HAI response after vaccination with LAIV, we considered only subjects vaccinated with TIV in further correlation analyses. There was a modest positive correlation between the number of IgG-secreting plasmablasts at day 7 and the HAI response at day 28 after vaccination (Fig. 1e). Because the frequency of plasmablasts returns to a barely detectable amount by day 14 after vaccination $^{15}$, this correlation suggested that the later antibody response was associated with early circulation of plasmablasts in the blood of vaccinees $^{3}$. However, given the modest correlation $(r=0.43)$, there was clearly a need for more robust correlates of immunogenicity.
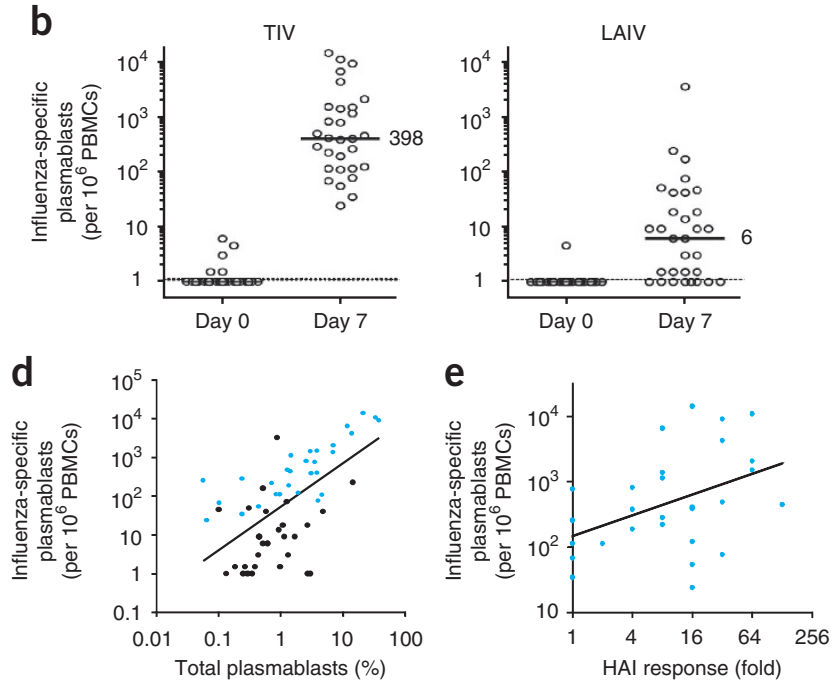

Figure 1 Analysis of humoral immunity to influenza vaccination. (a) HAI titers in plasma on day 28 after vaccination with TIV or LAIV, relative to baseline (day 0); results are the highest HAI response among all three influenza strains in the vaccine: Iow responders, no increase above twofold; high responders, fourfold or more above baseline. $P<0.0001$, mean HAI response, TIV versus LAIV ( $t$-test). (b) ELISPOT assay of influenza-specific IgG-secreting plasmablasts among PBMCs from all vaccinees at 0 and $7 \mathrm{~d}$ after vaccination. Each symbol represents an individual donor; small horizontal lines indicate the median (numbers adjacent median values); dotted lines are the limit of detection. (c) Flow cytometry analysis of plasmablasts in the plasmablast gate (CD3-CD20 ${ }^{\text {lo-neg} C D 19+C D 27 ~ h i ~ C D 38 ~}{ }^{\text {hi }}$ ) in blood from subjects vaccinated with TIV or LAIV. Numbers adjacent to outlined areas indicate percent cells in the plasmablast gate. (d) Frequency of plasmablasts, assessed by flow cytometry, versus the number of influenza-specific IgG-secreting plasmablasts, assessed by ELISPOT, at day 7 after vaccination with TIV (blue) or LAIV (black). $r=0.58$ (Pearson); $P<0.0001$ (for Pearson correlation; two-tailed test). (e) Influenza-specific IgG-secreting plasmablasts at day 7 versus the antibody response at day 28 after vaccination with TIV. $r=0.43$ (Pearson); $P=0.02$ (for Pearson correlation; two-tailed test). Data are from one experiment with 56 subjects assayed in duplicate (a), 61 subjects assayed in duplicate (b) or 59 subjects assayed once (c) or were generated from data in a-c (d,e). 

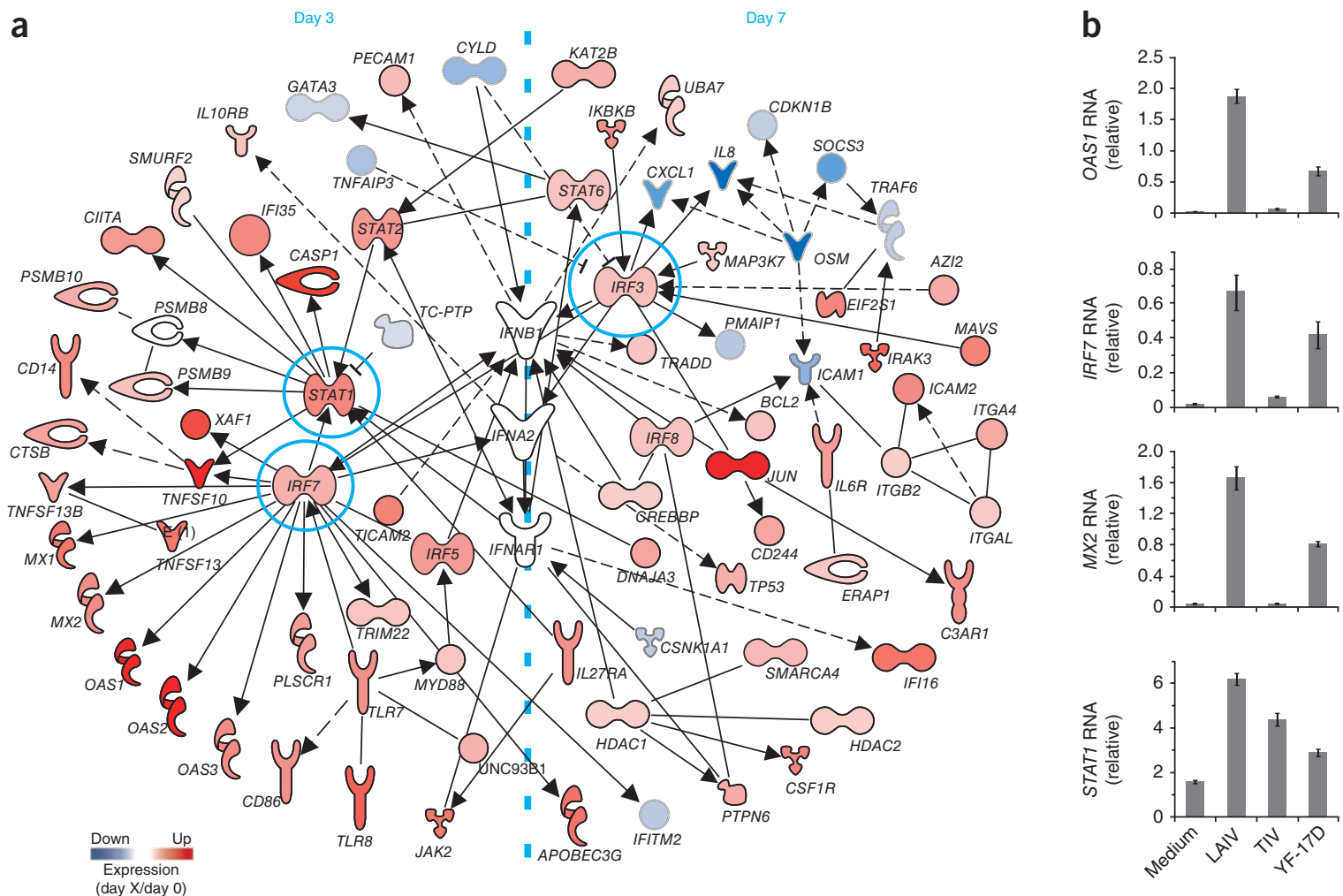

Figure 2 Molecular signature induced by vaccination with LAIV. (a) Interferon-related genes upregulated (Up) or downregulated (Down) on day 3 or 7 (' $X$ ' in key) after vaccination with LAIV relative to their expression at day 0 (colors in key): solid lines indicate direct interactions; dashed lines indicate indirect interactions. (b) Quantitative RT-PCR confirmation of the induction of key interferon-related genes (OAS1, IRF7, MX2 and STAT1) in PBMCs obtained from healthy subjects and left unstimulated (Medium) or stimulated for $24 \mathrm{~h}$ in vitro with LAIV, TIV or YF-17D; results are normalized to the expression of GAPDH (glyceraldehyde phosphate dehydrogenase) and are presented relative to those of unstimulated PBMCs. Data are representative of one experiment (a) or three independent experiments with one subject each (b; error bars, s.d.).

\section{Molecular signatures of influenza vaccines}

We first determined whether TIV and LAIV induced molecular signatures that were detectable in the blood. To identify such signatures of immunogenicity, we first measured by multiplex assay the concentrations of key cytokines in the plasma of vaccinees on days 0,3 and 7 after vaccination (Supplementary Fig. 2a). We selected ten cytokines or chemokines on the basis of their importance as key mediators of host immune responses (CCL5 (RANTES), interleukin $1 \alpha$, interferon- $\alpha 2$ (IFN- $\alpha 2$ ), CCL3 (MIP-1 $\alpha$ ), CCL11 (eotaxin), interleukin 12 subunit p70, IFN- $\gamma$, interleukin $1 \beta$, CXCL10 (IP-10) and CCL2 (MCP-1)). Among those, only the chemokine CXCL10 (IP-10) was significantly induced by TIV on day 3 relative to its expression on day $0(P=0.0189$ ( $t$-test); Supplementary Fig. 2b). None of those cytokines were significantly induced or repressed by vaccination with LAIV. The concentration of CXCL10 (IP-10) at day 3 relative to its baseline concentration was negatively correlated to the HAI response at day 28 after vaccination (Supplementary Fig. 2c), which suggested possible involvement of CXCL10 (IP-10) in the antibody response. However, the correlation coefficient was modest $(r=-0.48)$, which again emphasized the need for more robust correlates of immunogenicity.

To determine in an unbiased way the expression changes induced by vaccination against influenza on a genome-wide scale, we did microarray analysis using peripheral blood mononuclear cells (PBMCs) collected from all 56 vaccinees on days 0, 3 and 7 after vaccination. We calculated the change in expression by subtracting the $\log _{2}$ expression value at day 0 from its corresponding value day 3 or 7 , and we filtered out genes if we observed no increase or decrease greater than $25 \%$ (1.25-fold) in at least $20 \%$ of the vaccinees. After that step, we applied three independent statistical tests to the remaining genes and considered only genes identified by all three analyses as being differently expressed.

Transcriptome analysis of vaccinees showed that LAIV and TIV induced very different gene signatures (Supplementary Fig. 3a). However, the expression of 1,445 probe sets was altered similarly by both vaccines (Supplementary Fig. 3a). Among these common 'differentially expressed genes' (DEGs), ingenuity pathway analysis identified a network composed of several genes related to inflammatory and antimicrobial responses (Supplementary Fig. 3b; complete list of DEGs after vaccination with TIV or LAIV, Supplementary Table 1). This indicated that processes related to innate immunity may have influenced the immunogenicity of each vaccine. The expression of several interferon-related genes was altered after vaccination with LAIV but not after vaccination with TIV (Fig. 2). Type 1 interferons are central components of the innate immune response to virus ${ }^{16}$. Therefore, the higher expression of type I interferon-related genes may be attributed to the replication competence of LAIV. Our analysis identified genes encoding molecules closely associated with the interferon signaling pathways, such as STAT1, STAT2, TLR7, IRF3 and IRF7 (Fig. 2a). Notably, the difference in expression for many interferon-related genes was greatest at day 3 after immunization with LAIV (Fig. 2a).

We also compared the gene signatures of the two influenza vaccines with that of another live attenuated vaccine, the YF-17D vaccine against yellow fever ${ }^{6}$. For consistency with that publication ${ }^{6}$, we applied the same stringency and criteria to identify genes with differences in 
Figure 3 Molecular signatures induced by vaccination with TIV. (a) Heat map of gene signatures of cells of the immune response, identified by meta-analysis. Expression of each gene (rows) is presented as s.d. above (red) or below (blue) the average value for that gene for all samples (columns). mDC, myeloid DC; pDC, plasmacytoid DC; NK, natural killer. (b) Enrichment for genes upregulated by TIV among genes with high expression in any PBMC subset (numbers in plot indicate enrichment (fold)). (c) Enrichment for genes upregulated by TIV among genes with high expression in B cells and also in a specific B cell subset. (d) Heat map of genes upregulated after vaccination with TIV and also with high expression in B cells (PBMCs) and ASCs (B cell subsets); 'abParts' indicates probe sets mapping to antibody variable regions, and Affymetrix probe identifiers are provided for probe sets not annotated. (e) Enrichment for genes upregulated by LAIV among genes with high expression in any PBMC subset. ${ }^{*} P<10^{-10}$ (two-tailed Fisher's exact test). Data are representative of 28 experiments with 281 samples.

expression in subjects vaccinated with YF-17D, as follows: we filtered out genes if we found no increase or decrease in expression (on day 3 or 7 relative to baseline) greater than 1.41 -fold in at least $60 \%$ of the vaccinees; we used one-way analysis of variance with the Benjamini and Hochberg false-discovery-rate method with a cutoff of 0.05; and genes had to have a difference in expression in both YF-17D tri$a^{6}{ }^{6}$. However, this time we did the analysis at the level of the probe set instead of defining genes based on the UniGene database (National Center for Biotechnology Information). Although subjects vaccinated with YF-17D had a gene-expression profile distinct from that of those vaccinated against influenza, many interferon-related genes were commonly induced by YF-17D and LAIV (data not shown). RT-PCR analysis of RNA from PBMCs stimulated in vitro with LAIV, TIV or YF-17D confirmed that interferon-related genes were upregulated $24 \mathrm{~h}$ after treatment with LAIV or YF-17D but not after stimulation with TIV (Fig. 2b). Together these data demonstrated that vaccination with TIV or LAIV induced distinct molecular signatures in the blood.

\section{Molecular signatures of sorted cell subsets}

We did microarray analyses of the gene-expression profiles of PBMCs isolated from the blood of vaccinees at baseline and at days 3 and 7 after vaccination. One confounding variable here was that the observed transcriptional changes may have resulted from new induction of gene expression or may have simply reflected the changing cellular composition of the PBMC compartment. To overcome this issue, we used the approach of isolating and identifying the genomic signatures of each subset in the PBMC pool. We did microarray experiments with the following four different cell types, obtained from subjects vaccinated with LAIV $(n=6)$ or TIV $(n=6)$ and sorted by flow cytometry: CD $19^{+} \mathrm{B}$ cells, CD $14^{+}$monocytes, CD $11 \mathrm{c}^{\text {hi }} \mathrm{CD} 123^{\text {lo }}$ myeloid dendritic cells (DCs) and CD123 ${ }^{\text {hi }}$ CD $11 c^{\text {lo }}$ plasmacytoid DCs. We extracted, amplified and labeled total RNA from 96 sorted cell samples at baseline and day 7 and hybridized the RNA on microarray chips (Supplementary Fig. 4a). We did significance analysis of microarrays ${ }^{17}$ for each subset, separately comparing the values at day 7 with the corresponding baseline values. This approach identified hundreds to thousands of probe sets with differences in expression after vaccination with TIV or LAIV (Supplementary Fig. 4b and Supplementary Table 2), which demonstrated that vaccines against influenza produced global expression changes for each cell type.

In subjects vaccinated with TIV, myeloid DCs and B cells had the most DEGs (Supplementary Fig. 4b). Notably, there was an enrichment for DEGs associated with plasmablasts (Supplementary Fig. 4c). However, because a substantial proportion of plasmablasts die after being frozen and thawed (data not shown), the DEGs observed in the
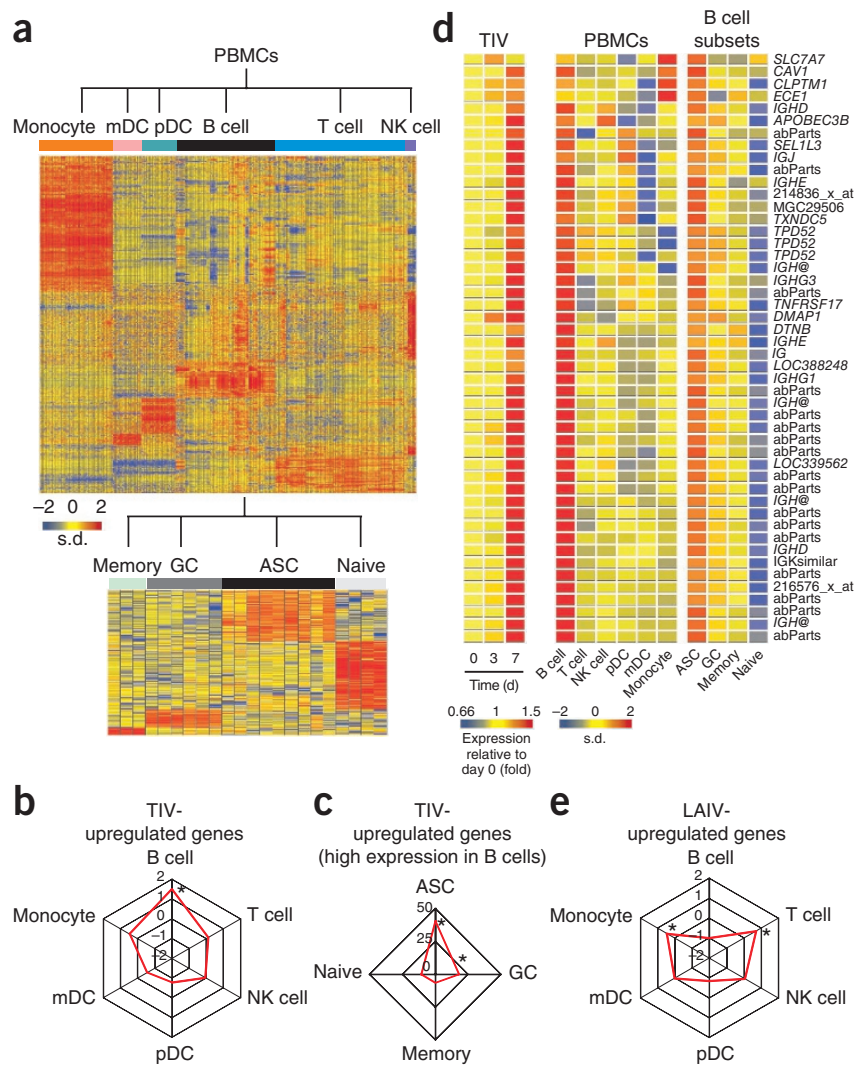

B cell compartment were probably an underestimation of the DEGs associated with plasmablasts (Fig. 3). Nevertheless, we were still able to identify DEGs related to antibody-secreting cells (ASCs) and the unfolded protein response in sorted B cells after immunization with TIV (Supplementary Fig. 4c,d). To cope with the large amount of immunoglobulin proteins that are produced, ASCs must greatly increase the function of their secretion machinery, which may lead to the accumulation of misfolded proteins in the endoplasmic reticulum ${ }^{18,19}$. In response to such stress, the cells activate intracellular signal-transduction pathways and the unfolded protein response, which protects the cells by enhancing the capacity of the secretory apparatus and by diminishing the endoplasmic reticulum load ${ }^{20}$. After vaccination with TIV, upregulation of genes encoding two transcription factors, XBP-1 and ATF6B, which are central orchestrators of the unfolded protein response, was detectable in sorted B cells but not in PBMCs (Supplementary Fig. 4d).

In subjects vaccinated with LAIV, in contrast to results obtained with those vaccinated with TIV, the plasmacytoid DC subset generated the most DEGs (Supplementary Fig. 4b). Of the many interferon-related genes induced by LAIV (Fig. 2a), we found that 37 were induced in at least one subset of the sorted cells. Of those, 17 and 14 were upregulated in monocytes and plasmacytoid DCs, respectively (Supplementary Fig. 4e). In addition, there were 44 interferon-related genes that were induced or repressed in at least one subset of the sorted cells but not in the PBMCs (Supplementary Fig. 4e). Most were upregulated in myeloid and plasmacytoid DCs (Supplementary Fig. 4e). These data suggest that antigen-presenting cells may be important in the innate response to vaccination with LAIV. The large number of interferon-related genes 'missing' from the PBMC analysis may have been due to the fact that myeloid DCs and plasmacytoid DCs together represent $<1 \%$ of total PBMCs ${ }^{21}$.

The observations reported here indicated the type of information that can be obtained by examination of the gene-expression profiles 
of sorted cell types. However, evaluating the gene-expression signatures of individual subsets of cells isolated by flow cytometry presents a considerable challenge. The practical use of such an approach is very limited, both logistically (that is, the need to use freshly isolated samples to prevent the 'preferential' loss of certain cell types, such as plasmablasts and effector T cells) and financially (that is, the need for large numbers of gene chips). Therefore, as described below, we devised an alternative strategy.

\section{Meta-analysis of cell type-specific signatures}

Human PBMCs consist of many different cell types, each with a distinct transcriptome. A published study has demonstrated the use of a deconvolution method to analyze cell type-specific gene expression differences in complex tissues ${ }^{22}$. We devised an independent strategy to discern cell type-specific transcriptional signatures with the results of the PBMC microarray analyses. We did a meta-analysis of publicly available microarray studies in which the gene-expression profiles of isolated individual cell types of PBMCs (such as T cells, $\mathrm{B}$ cells, monocytes, natural killer cells and so on) or B cell subsets (such as naive, memory and germinal center B cells and ASCs from blood or tonsils) had been analyzed (Supplementary Fig. 5a,b). To avoid issues of cross-platform normalization and probe selection, we used only samples hybridized to Affymetrix Human Genome U133 Plus 2.0 Arrays or Affymetrix Human Genome U133A Arrays in our meta-analysis. Additionally, for each study, we manually removed samples based on the severity of the disease or treatment and/or the method of cell purification (samples and studies, Supplementary Table 3). We included in our meta-analysis microarray data of flow cytometry-sorted plasmacytoid and myeloid DCs obtained from PBMCs of subjects before and after vaccination with TIV or LAIV (Supplementary Fig. 4a). We compared the expression profile of a given cell subset with the expression profile of all other subsets by $t$-test $(P<0.05$; mean change, over twofold). We designated a gene as having high expression in a particular cell type by determining the number of times the gene was upregulated in the cell type by all possible pairwise comparisons with its expression in other cell types (Supplementary Fig. 5b and Supplementary Methods). We then compared the genomic signatures of cells of the immune response obtained by this approach (Fig. 3a and Supplementary Table 4) with the genomic signatures of subjects vaccinated against influenza.

Our meta-analysis confirmed that the group of genes upregulated by TIV was enriched for genes with high expression in B cells (Fig. 3b) and, among those, genes with high expression in ASCs (Fig. 3c). We prepared a heat map of the genes upregulated in ASCs after vaccination with TIV (Fig. 3d). Among the genes upregulated were those encoding 'antibody parts' (rearranged variable-diversity-joining immunoglobulin gene segments) and several other genes encoding parts of immunoglobulins (IGH@, IGHE, IGHG3, IGHG1 and IGHD), as well as TNFRSF17 (which encodes BCMA, a member of the BAFF-BLyS family of receptors ${ }^{23}$, and whose expression has been shown before to be a key feature of the best predictive signatures of neutralizing antibody responses to $\mathrm{YF}-17 \mathrm{D}^{6}$ ). These results confirmed the results obtained by flow cytometry and ELISPOT, with which we observed a greater frequency of $\operatorname{IgG}^{+}$and $\operatorname{IgA}^{+}$ASCs in the blood of vaccinees at day 7 after vaccination with TIV (Fig. 1 and Supplementary Fig. 1).

In addition to the ASC signature, we observed a signature composed of several genes encoding molecules that orchestrate the unfolded protein response ${ }^{19,20}$ (data not shown). The large number of XBP-1 target genes with differences in expression after vaccination was consistent with a role for XBP-1 in orchestrating the differentiation of plasma cells ${ }^{19}$. Among those, genes such as ATF6, MANF, CREB3, PDIA4, DNAJB11, HSP90B1, HERPUD1 and $D N A J B 9$ encode molecules are already known to be involved in the unfolded protein response ${ }^{24-26}$.

In contrast to results obtained for TIV, analysis of the transcriptional signature induced by LAIV by meta-analysis showed considerable enrichment for genes with high expression in $\mathrm{T}$ cells and monocytes (Fig. 3e). We also found many genes with high expression in natural killer cells, although these results did not reach statistical significance (data not shown). Among the interferon-related genes upregulated after vaccination with LAIV (Fig. 2a), most had high expression in monocytes and natural killer cells (data not shown). That result was similar to our microarray analysis of flow cytometrysorted cells obtained from subjects vaccinated with LAIV, in which most interferon-related genes with differences in expression in PBMCs and at least one cell subset had high expression in monocytes (Supplementary Fig. 4e). These results indicate that the innate immune responses can have an important role in the mechanism of action of this live attenuated virus vaccine.

\section{Signatures that correlate with the antibody response}

Vaccination with TIV induced considerable variation in the magnitude of the HAI response (Fig. 1a). To gain insight into the potential mechanisms underlying that variation and to identify gene signatures with which we could predict the magnitude of the HAI response, we searched for early gene signatures that correlated with the B cell responses at days 7 and 28 after vaccination with TIV (complete list, Supplementary Table 5). Pearson correlation analysis identified 600-1,100 probe sets that correlated, either directly or inversely, with the magnitude of the HAI response (Fig. 4a). Among those were several genes known to be regulated by XBP-1 and to be involved in the differentiation of plasma cells and the unfolded protein response (Fig. $\mathbf{4 b}$ ).

Ingenuity pathway analysis of the genes that were either positively or negatively correlated with HAI titers showed enrichment for genes related to the cell-mediated immune response and to the infection mechanism and inflammatory response, respectively (Supplementary Fig. 6a,b). The identification of genes such as TLR5, CASP1, PYCARD, NOD2 and NAIP suggested previously unknown mechanistic links between host innate immunity and humoral responses to influenza vaccination. In fact, research has shown that a candidate vaccine against influenza composed of a recombinant fusion protein linking influenza antigens to the Toll-like receptor 5 ligand flagellin may induce potent immunogenicity in mice $^{27}$ and humans ${ }^{28,29}$. In addition, canonical pathways, such as $\mathrm{T}$ cell receptor antigen receptor signaling and CTLA-4 signaling in cytotoxic T lymphocytes, included many of the genes present in the cell-mediated immune response network and were among those with the highest enrichment score by ingenuity pathway analysis (Supplementary Fig. 7a,b). Although further experimentation is needed, these data indicated a possible association between cellular responses and humoral responses to vaccination with $\mathrm{TIV}^{30}$. Among the top canonical pathways enriched for genes positively correlated to HAI response (Supplementary Fig. 7c), we found networks associated with innate immunity, such as the natural killer cell signaling network, and network for the production of nitric oxide and reactive oxygen species in macrophages (Supplementary Fig. 7d). Our analysis also showed that the expression of interferonrelated genes (including those encoding the receptors for interferon- $\alpha$ and interferon- $\gamma$ ) on day 3 after vaccination was correlated to the HAI response (Supplementary Fig. 8), which suggested a link between the interferon response and the antibody response ${ }^{31}$. 
Figure 4 Molecular signatures that correlate with titers of antibody to TIV. (a) Heat map of probe sets (rows) and subjects (columns) whose baseline-normalized expression at day 3 (top) or day 7 (bottom) correlated with baseline-normalized antibody response at day 28 after vaccination with TIV (colors in map indicate gene expression at day 3 or 7 relative to expression at day 0). Right margin, number of probe sets with negative correlation (blue) or positive correlation (red). Probe sets that correlated with the HAI response on both day 3 and day 7 were considered 'day 7'. $P<0.05$ (Pearson). (b) HAI response-correlated genes associated with the unfolded protein response (purple shading) or ASC differentiation (tan shading) and/or regulated by XBP-1 (solid and dashed lines as in Fig. 2a). $P<0.05$ (Pearson). (c) Enrichment for genes (among those with high expression in any PBMC subset) whose expression on day 3 or 7 after vaccination with TIV was positively or negatively correlated with HAI titers (cutoff, $P<0.05$ (Pearson)).

${ }^{*} P<10^{-10}$ (two-tailed Fisher's exact test).

(d) Heat map of probe sets with high expression in B cells and ASCs whose baseline-normalized expression correlated with the baseline-

normalized HAI response. $P<0.05$ (Pearson). Data are representative of one experiment with 28 subjects.

Next we compared the genes whose expression correlated with the HAI response at day 28 after vaccination of subjects with TIV with the genomic signatures of the cells of the immune response defined by our metaanalysis. This approach showed that the set of genes positively correlated to HAI response was enriched for genes with high expression in B cells (Fig. 4c) and, more specifically, in the ASC subset (Fig. 4d). The genes with negative correlation to the HAI response were substantially enriched among the genes with high expression in $\mathrm{T}$ cells (Fig. 4c), which supported the identification of the T cell pathways by ingenuity pathway analysis (Supplementary Fig. 7a,b). Together these data demonstrated the identification of early signatures that correlated with later HAI titers induced by TIV.

\section{Molecular signatures to predict antibody responses}

Once we had delineated signatures that correlated with the magnitude of HAI response, our next step was to identify the minimum sets of genes we could use to predict such a response. Ideally, such sets of genes must be able to be used to accurately classify high responders versus low responders in additional and independent TIV trials. For this, we used DAMIP (discriminant analysis via mixed integer programming ${ }^{32,33}$ ), which is a very powerful supervised-learning classification method for predicting various biomedical and 'biobehavioral' phenomena ${ }^{32,33}$.

In initial analyses, we classified the subjects vaccinated with TIV into two 'extreme' groups: very low HAI responders, and very high HAI responders. The former group consisted of subjects with an increase of twofold or less in HAI titers against any of the three influenza strains of the vaccine (Fig. 1a). The latter group consisted of subjects with an increase of eightfold or more in the HAI response for

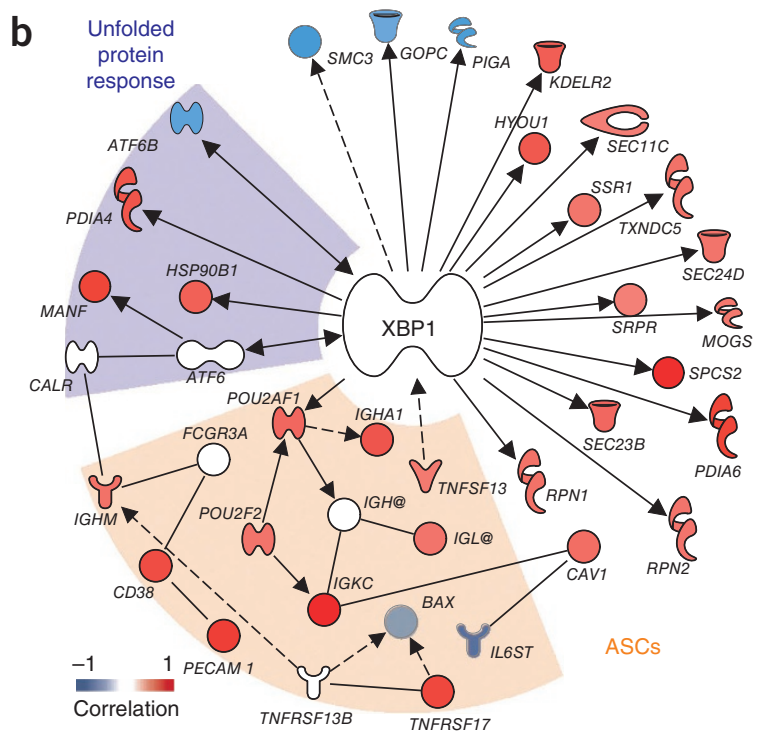

C
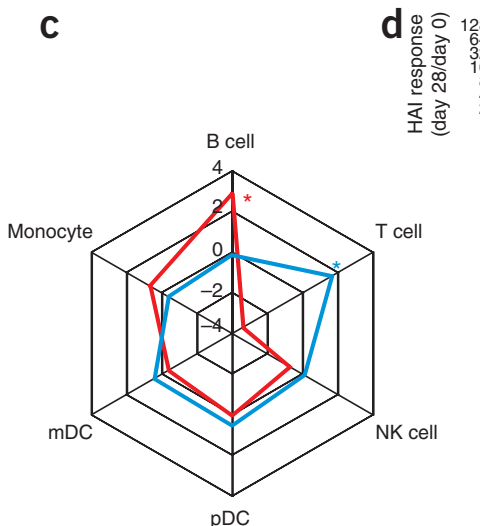

HAl response at day 28 - Positive correlation (day 7) - Negative correlation (day 7)

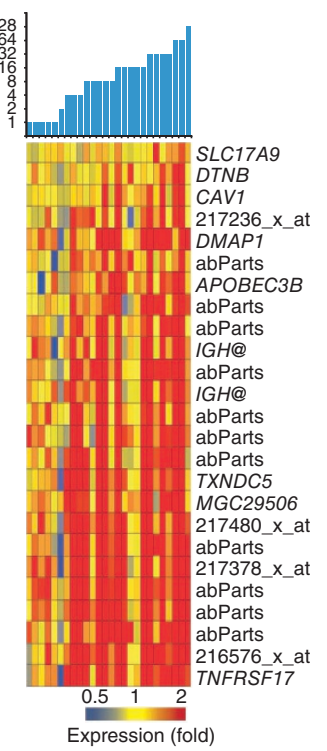

at least one of the three influenza strains of the vaccine. We did not analyze subjects with intermediate HAI response (between twofold and eightfold) and subjects for whom microarray data were not available at either day 3 or day 7 after vaccination $(n=7)$. We used that trial (the 2008-2009 trial) to train the DAMIP model to establish an unbiased estimate of correct classification. We used a second, independent trial to evaluate the predictive accuracy of the classification rules identified in the first trial (Fig. 5a). The second trial (the 2007-2008 trial) consisted of the microarray gene-expression profiles of subjects $(n=9)$ vaccinated with TIV in the previous year. With this approach, DAMIP model identified 12 sets of genes containing two to four genes each (each set associates with one predictive rule) from 2008-2009 trial with a tenfold cross-validation accuracy over $90 \%$. The resulting 'blind prediction' accuracy of the 2007-2008 trial (predicting low or high responders) was over $90 \%$. Furthermore, some of the 271 sets of discriminatory genes offered an accuracy of over $90 \%$ in both tenfold cross-validation in the training trial and 'blind prediction' accuracy (Fig. 5a and Supplementary Table 6).

We then used real-time RT-PCR to confirm that 44 genes from the DAMIP gene signatures encoded molecules with potential biological relevance and/or utility as a predictor of influenza 
Figure 5 Signatures that can be used to predict the antibody response induced by TIV.

(a) Experimental design used to identify the early gene signatures that can be used to predict antibody responses to vaccination with TIV: the 2008-2009 trial was used as a training set to identify predictive signatures with the DAMIP model; those signatures were then tested on the data from the 2007-2008 trial (the testing set). The expression of a subset of genes in the DAMIP predictive signatures of the 2007-2008 and 2008-2009 trials was then quantified by RT-PCR in a third independent trial (2009-2010 trial); the DAMIP model was again used to confirm the predictive signatures. (b) RT-PCR confirmation of the expression of a subset of genes in the predictive signatures generated by the DAMIP model. Each symbol represents a single gene at a given time point. $P<10^{-11}$, microarray versus RT-PCR (Pearson); $r=0.68 ; n=2,897 \mathrm{XY}$ pairs. Data are representative of one experiment with 44 genes from 28 subjects at two time points. (c) DAMIP gene signatures identified with the 2008-2009 trial as the training set and the 2007-2008 and 2009-2010 trials as the validation sets (DAMIP model 3); the accuracy represents the number of subjects correctly classified as 'low responders' or 'high responders' (Fig. 1a). Data are representative of three independent experiments.

vaccine immunogenicity. We found a significant positive correlation $\left(r=0.679 ; P=3.25 \times 10^{-12}\right)$ for changes in expression on day 3 or 7 relative to baseline expression as detected by microarray and RT-PCR (Fig. 5b and Supplementary Table 7), which confirmed the correctness of the microarray data. More notably, that result gave us confidence to test some of the candidate predictors of immunogenicity in a third and independent influenza vaccine trial (Fig. 5a). We collected RNA from PBMCs of subjects $(n=30)$ vaccinated with TIV during the 2009-2010 influenza season and analyzed this RNA by real-time RT-PCR. We then used the expression of the 44 genes selected from the initial DAMIP gene signatures to confirm their utility in predicting the magnitude of antibody response in this third TIV trial (Fig. 5a). To avoid the identification of 'over-trained' rules, we re-ran the DAMIP analysis using the 2008-2009 trial as the training set and the 2007-2008 and 2009-2010 trials as the blind predictive sets. This approach identified 47 sets of genes; some of these we used to correctly classify $>85 \%$ of the vaccines as being very low HAI responders or very high HAI responders in any of the three trials (Supplementary Table 8).

Because seroconversion after vaccination is widely defined as a fourfold increase in HAI titers ${ }^{34}$, we ran an additional DAMIP analysis using a cutoff of fourfold to classify the vaccinees (Fig. 5a). Thus, we classified subjects with an increase of fourfold or greater in the HAI titers after vaccination as 'high responders' and those with an increase of twofold or less as 'low responders'. With the 2008-2009 trial as a training set and 2007-2008 and 2009-2010 trials as blind predictive sets, the DAMIP model generated 42 sets of gene signatures (Fig. 5c), each composed of three to four discriminatory genes, some of which had an unbiased estimate of correct classification above $85 \%$, as determined by tenfold cross-validation and blind prediction (Supplementary Table 9). One of the genes present in the DAMIP gene signatures, TNFRSF17, was also identified in DAMIP models used to predict antibody responses to vaccination with YF-17D ${ }^{6}$.

Among the genes in the TIV DAMIP models, we found five members of the leukocyte immunoglobulin-like receptor family (Supplementary Table 9). These genes are expressed by immuneresponse cells of both myeloid and lymphoid lineages and the molecules they encode are thought to have an immunomodulatory role in the innate and adaptive immune systems by regulating $\mathrm{T}$ cells and autoimmunity ${ }^{35-37}$. Our meta-analysis showed that these genes had high expression in monocytes and myeloid DCs at day 3 after vaccination (data not shown). These results and the presence of five members of this family among markers of antibody responses to influenza vaccination raised the possibility of previously unknown roles for these innate immune receptors in regulating antibody responses.

\section{CaMKIV regulates the antibody response}

To demonstrate that the gene signatures identified in our study could be used to generate new hypotheses, we selected one gene in the predictive signature, CAMK4, for functional confirmation experiments. CaMKIV is involved in several processes of the immune system, such as $\mathrm{T}$ cell development ${ }^{38-40}$, inflammatory responses ${ }^{41,42}$ and the maintenance of hematopoietic stem cells ${ }^{43}$. However, nothing is known about the possible role of CaMKIV in B cell responses.

The change in CAMK4 expression on day 3 after vaccination with TIV was negatively correlated with the antibody response on day 28 after vaccination in two independent trials (Fig. 6a). Additionally, the change in $C A M K 4$ expression was negatively correlated with the population expansion of IgG-secreting plasmablasts at day 7 (Fig. 6b), which suggested a possible role for CaMKIV in the regulation of antibody responses to vaccination against influenza.

In vitro stimulation of mouse splenocytes with TIV resulted in phosphorylation of CaMKIV (Fig. 6c), which suggested that this vaccine may trigger activation of CaMKIV. That finding was further demonstrated in human PBMCs, in which in vitro stimulation with influenza vaccine resulted in phosphorylation of CaMKIV as early as $2 \mathrm{~h}$ after stimulation (Fig. 6d). The mechanism by which this occurs remains to be identified.

To check if CaMKIV regulates the antibody response to influenza

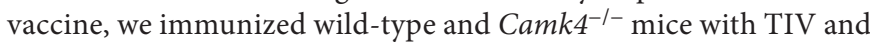
measured serum concentrations of IgG1 and IgG2c on days 7, 14 and 28 after vaccination (Fig. 6e). After immunization, $\mathrm{Camk}^{-1-}$ mice had a significantly greater antibody response than that of wild-type mice (Fig. 6e). The biggest difference was on day 7, with 3- to 6.5-fold higher antibody titers in $\mathrm{Camk4}^{-/-}$mice than in wild-type mice (Fig. 6e). These results supported our prediction based on the microarray results and suggested that CaMKIV is important in the regulation of B cell response. 
a

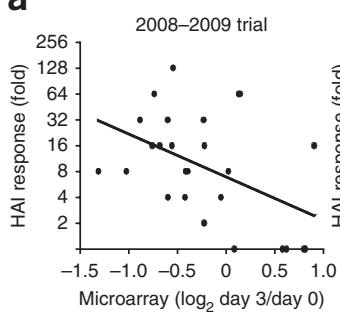

b

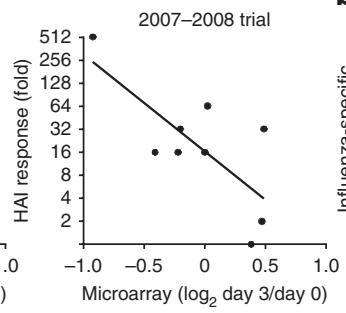

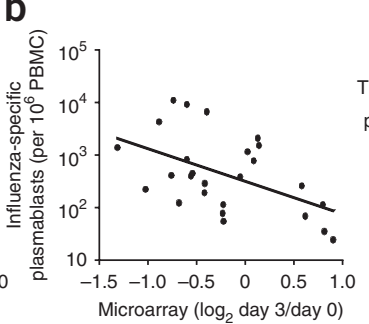

c

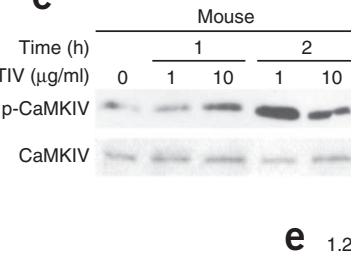

d

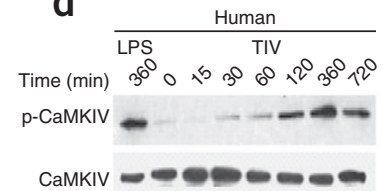

Figure 6 CaMKIV regulates the antibody response to vaccines against influenza. (a) HAI response at day 28 versus microarray analysis of CAMK4 mRNA in PBMCs at day 3 after vaccination with TIV in the 2008-2009 trial (left; $r=-0.47$ (Pearson); $P=0.016$ (for Pearson correlation; two-tailed test) or the 2007-2008 trial (right; $r=-0.73$ (Pearson); $P=0.024$ (for Pearson correlation; two-tailed test). (b) ELISPOT analysis of influenza-specific lgG-secreting plasmablasts at day 7 versus microarray analysis of CAMK4 mRNA on PBMCs at day 3 after vaccination with TIV. (c) Immunoblot analysis of the phosphorylation (p-) of mouse CaMKIV after in vitro stimulation of splenocytes for 1 or $2 \mathrm{~h}$ with various doses of TIV (above lanes).

(d) Immunoblot analysis of the phosphorylation of CaMKIV after in vitro stimulation of human PBMCs for 0-720 min with lipopolysaccharide (LPS) or TIV. (e) Serum antigen-specific IgG1 (top) and IgG2c (bottom) responses of wild-type and $\mathrm{Camk}^{-/-}$mice at days 7, 14 and 28 after immunization with TIV (symbols represent individual mice), presented as absorption at $450 \mathrm{~nm}\left(A_{450}\right) .{ }^{*} P<0.05$ and ${ }^{* *} P<0.01$ (Student's $t$-test). Data are representative of one trial each with 26 subjects (2008-2009) or 9 subjects (2007-2008; a), one experiment with 26 subjects (b), three experiments (c,d) or at least four independent experiments (e).
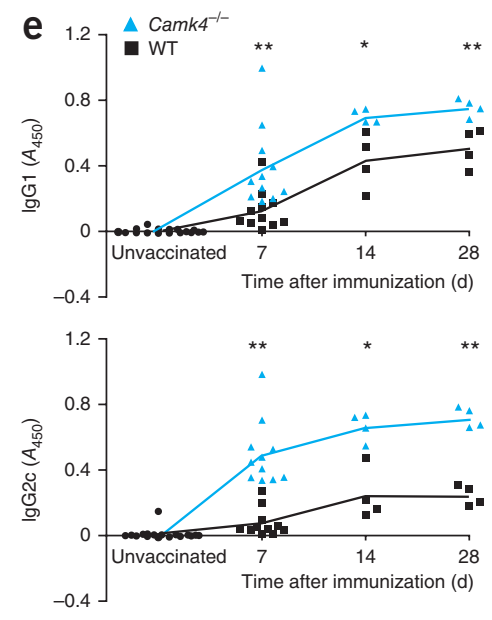

\section{DISCUSSION}

Despite the great success of vaccines, little is understood about the mechanisms by which effective vaccines stimulate protective immune responses. Two developments are beginning to offer such understanding: increasing appreciation of the key roles of the innate immune system in sensing vaccines and tuning immune responses, and emerging advances in systems biology 44 . A systems biology approach has been used to obtain a global picture of the immune responses in humans to vaccine YF-17D against yellow fever, one of the most successful vaccines ever developed. This approach has identified unique biomarkers (molecular signatures) used to predict the magnitude of the antigen-specific $\mathrm{CD} 8^{+} \mathrm{T}$ cell and antibody responses induced by YF-17D ${ }^{6,7}$ and has resulted in the formulation of new hypotheses about the mechanism of action of this vaccine. However, whether such an approach could have broad utility in the identification of signatures of immunogenicity of other kinds of vaccines, particularly inactivated vaccines, and whether such signatures would be informative about the underlying mechanisms of immunity remain unknown. To address these issues, we did a series of studies over three consecutive influenza seasons. The goal of these studies was to analyze in detail the innate and adaptive immune responses to vaccination with two vaccines against influenza, TIV and LAIV, to identify early molecular signatures that can be used to predict later immune responses and to obtain insight into the mechanisms that underlie immunogenicity. According to guidelines established by the US Food and Drug Administration ${ }^{14}$, seroconversion can be defined as an HAI titer of 1:40 or more and a minimum increase of fourfold in antibody titer after vaccination. However, it often takes several weeks after vaccination to achieve this titer; therefore, the ability to predict seroconversion just a few days after vaccination and identify nonresponders would be of great value from a public health perspective. We thus used systems biology approaches to identify early signatures that we used to predict HAI titers 4 weeks after vaccination. To accomplish this goal, we used an interdisciplinary approach, including gene-expression profiling by microarray, RT-PCR and computational methods, combined with cellular and molecular biological approaches, as well as experiments involving genetically deficient mice. Our data have demonstrated that such a systems biology approach can indeed be used not only to identify predictive signatures but also to obtain new insights about the immunological mechanisms involved.

Although the clinical effectiveness of both vaccines is similar, LAIV induces lower serum antibody response in adults than does $\operatorname{TIV}^{1,3,45}$. This probably reflects the lower 'take' of LAIV because of preexisting mucosal IgA that can neutralize the virus ${ }^{13}$. Nevertheless, our microarray analysis identified a large number of genes with differences in expression, most related to the type I interferon response, in the PBMCs of subjects vaccinated with LAIV. Future studies should focus on analyzing changes in the transcriptome of the nasal mucosa after vaccination with LAIV and how that correlates with or can be used to predict local antibody responses.

Among the genes induced by vaccination with TIV, we found enrichment for genes with high expression in ASCs. This result may have reflected the rapid proliferation of plasmablasts at day 7 after vaccination ${ }^{15}$; however, our microarray analysis of B cells sorted from subjects vaccinated against influenza indicated that the changes in expression observed in PBMCs could also have been derived from real transcriptional changes in B cells. The transcription factor XBP-1, which is essential for the differentiation of ASCs and the unfolded protein response ${ }^{18}$, and its target genes were upregulated after vaccination with TIV and correlated with IgG and HAI responses. The genes identified by our study may offer new opportunities for studying the complex mechanisms involved in the unfolded protein response and its link to ASC differentiation ${ }^{18}$.

A key question was whether the signatures that can be used to predict the $\mathrm{T}$ cell and $\mathrm{B}$ cell response to one vaccine can also be used to predict such responses to another vaccine. Notably, of the 133 genes present in the 271 DAMIP gene signatures that we used to predict the antibody response to vaccination with TIV, 7 were also predictors of the antibody response to vaccination with the YF-17D vaccine against yellow fever ${ }^{6}$. Key genes in the predictive signatures were TNFRSF17, which encodes BCMA, a receptor for the B cell growth factor BLyS (known to have a key role in $\mathrm{B}$ cell differentiation ${ }^{23}$ ), and $C D 38$, which encodes a surface protein important in lymphocyte development ${ }^{46,47}$. BCMA belongs to a family of molecules (BAFF, APRIL, BAFF-R and $\mathrm{TACI}$ ) that regulate the differentiation of plasma cells and antibody 
production $^{23}$. Notably, there were strong correlations between the expression of genes encoding APRIL, BAFF-R and TACI and the magnitude of the HAI titers in response to vaccines against influenza and the magnitude of neutralizing antibody response to YF-17D (data not shown), which suggested that this network may be critically involved in regulating antibody responses to different vaccines. The functional relevance of this network in mouse models remains to be determined. It also remains to be seen whether this network represents a common predictor of antibody responses induced by many vaccines.

A second issue was whether the data generated from such studies would be useful in providing new biological insights into the regulatory mechanisms that underlie vaccine immunogenicity. Our experiments with $\mathrm{Camk}^{-/-}$mice demonstrated that such data can indeed identify unexpected biological targets, which can be mechanistically confirmed by mouse models. Although the data demonstrated a potent role for CaMK4 in regulating antibody responses to vaccines against influenza, further work is needed to delineate the cellular mechanisms involved.

Third, whether signatures that can be used to predict immunogenicity can also be used to predict efficacy must be considered. Several studies have shown that serum HAI antibody concentrations correlate with protection against influenza ${ }^{48-50}$. Seroconversion after vaccination, commonly defined as an increase of fourfold in HAI titers ${ }^{34}$, represents a useful surrogate for vaccine efficacy when applied to a population. However, this parameter may not provide the optimal prediction of protection in an individual vaccinee or a group of vaccinees. In addition, protective concentrations of antibody may vary according to the prevalent virus subtype and laboratory doing the assay ${ }^{51}$. Therefore, we used a more stringent parameter (an increase of eightfold or more in HAI response) to classify subjects with very high antibody responses. Using this cutoff in our analyses, the DAMIP method was able to identify gene signatures that we could use to predict the antibody response induced by vaccination with TIV. We confirmed the validity of these gene signatures in three independent trials, which demonstrated the robustness of our approach. To meet the definition of seroconversion in the US Food and Drug Administration Guidance for Industry document for this field (an HAI titer of 1:40 or more and a minimum increase of fourfold in antibody titer after vaccination $)^{14}$, we re-ran the DAMIP analysis using an increase of fourfold as a cutoff for defining high HAI responders. Again, the DAMIP method was able to identify sets of three to four discriminatory genes with an unbiased estimate of correct classification up to $90 \%$ for the three influenza trials. However, the generality of our findings in terms of using gene signatures in PBMCs to predict the immunogenicity and/or efficacy of other vaccines such as mucosal vaccines must be tested. It is likely that different signatures could be generated by analysis of mucosal tissues.

Finally, although the main goal of our study was a proof-of-concept demonstration of the feasibility of this approach in predicting vaccine immunogenicity, (rather than a demonstration of cost effectiveness), in ascertaining the predictive value of our signature in the 2009-2010 trial, we used a PCR-based assay (instead of an assay with gene-expression chips) of only a handful of genes. This demonstrated the feasibility of designing a cost-effective, PCR-based 'vaccine chip' that can be used to predict the immunogenicity of vaccines. Thus, we have shown how systems biology approaches can be applied to elucidate the molecular mechanisms of influenza vaccines. We envision that the predictive signatures of influenza vaccine-induced antibody responses may have implications in vaccine development, in the monitoring of suboptimal immune responses (in the elderly, infants or immunocompromised populations) or perhaps in identifying new correlates of protection.

\section{METHODS}

Methods and any associated references are available in the online version of the paper at http://www.nature.com/natureimmunology/.

Accession codes. GEO: microarray data, GSE29619.

Note: Supplementary information is available on the Nature Immunology website.

\section{ACKNOWLEDGMENTS}

We thank B.T. Rouse and R. Compans for discussion and comments on the manuscript, and $\mathrm{H}$. Oluoch for technical assistance. Supported the US National Institutes of Health (U19AI090023, HHSN266200700006C, U54AI057157, R37AI48638, R01DK057665, U19AI057266 and N01 AI50025 for the B.P. laboratory; AI30048 and AI057266 for the R.A. laboratory; DK074701 for the A.R.M. laboratory; Intramural Research Program of the National Institute of Allergy and Infectious Diseases for the K.S. laboratory; and UL1 RR025008 from the Clinical and Translational Science Award program, National Center for Research Resources for clinical work), the Bill \& Melinda Gates Foundation (Collaboration for AIDS Vaccine Discovery 38645 to the R.A. and B.P. laboratories), the National Science Foundation (E.K.L. laboratory) and the Centers for Disease Control (E.K.L. laboratory)

\section{AUTHOR CONTRIBUTIONS}

H.I.N. did all the experiments and analyses in Figures 2-6 and Supplementary Figures 2-8; J.W., G.-M.L., M.M. and V.K. did the analyses in Figure 1 and Supplementary Figure 1; E.K.L. did the DAMIP model analyses in Figure 5; L.R., A.R.M., S.P.K. and N.K. did the mouse experiments in Figure 6; W.N.H. helped with the microarray analyses in Supplementary Figure 4; S.L. assisted with the bioinformatics analyses of the data in Figure 3; A.A. did the microarray analysis of samples from the 2007 influenza annual season; S.M.-K., K.E.K., R.E. and A.K.M. assisted with the collection and processing of samples; K.S. measured HAI titers; R.A. helped conceive of and design the study and supervised the studies in Figure 1 and Supplementary Figure 1; B.P. conceived of the study and designed and supervised the experiments and analyses in Figures 1-6 and Supplementary Figures 1-8; and B.P. and H.I.N. wrote the paper.

\section{COMPETING FINANCIAL INTERESTS}

The authors declare no competing financial interests.

Published online at http://www.nature.com/natureimmunology/. Reprints and permissions information is available online at http://www.nature.com/ reprints/index.html.

1. Sasaki, S. et al. Comparison of the influenza virus-specific effector and memory B-cell responses to immunization of children and adults with live attenuated or inactivated influenza virus vaccines. J. Virol. 81, 215-228 (2007).

2. Fiore, A.E. et al. Prevention and control of influenza with vaccines: recommendation of the Advisory Committee on Immunization Practices (ACIP), 2010. MMWR Recomm. Rep. 59, 1-62 (2010).

3. Sasaki, S. et al. Influence of prior influenza vaccination on antibody and B-cell responses. PLoS ONE 3, e2975 (2008).

4. Zeman, A.M. et al. Humoral and cellular immune responses in children given annual immunization with trivalent inactivated influenza vaccine. Pediatr. Infect. Dis. J. 26 107-115 (2007).

5. Pulendran, B., Li, S. \& Nakaya, H.I. Systems vaccinology. Immunity 33, 516-529 (2010).

6. Querec, T.D. et al. Systems biology approach predicts immunogenicity of the yellow fever vaccine in humans. Nat. Immunol. 10, 116-125 (2009).

7. Gaucher, D. et al. Yellow fever vaccine induces integrated multilineage and polyfunctional immune responses. J. Exp. Med. 205, 3119-3131 (2008).

8. Pulendran, B. Learning immunology from the yellow fever vaccine: innate immunity to systems vaccinology. Nat. Rev. Immunol. 9, 741-747 (2009).

9. Monath, T.P. Yellow fever vaccine. Expert Rev. Vaccines 4, 553-574 (2005).

10. Querec, T. et al. Yellow fever vaccine YF-17D activates multiple dendritic cell subsets via TLR2, 7, 8, and 9 to stimulate polyvalent immunity. J. Exp. Med. 203, 413-424 (2006).

11. Barrett, A.D. \& Teuwen, D.E. Yellow fever vaccine-how does it work and why do rare cases of serious adverse events take place? Curr. Opin. Immunol. 21, 308-313 (2009).

12. Johnson, P.R. Jr. Feldman, S., Thompson, J.M., Mahoney, J.D. \& Wright, P.F. Comparison of long-term systemic and secretory antibody responses in children given live, attenuated, or inactivated influenza A vaccine. J. Med. Virol. 17 325-335 (1985).

13. Beyer, W.E., Palache, A.M., de Jong, J.C. \& Osterhaus, A.D. Cold-adapted live influenza vaccine versus inactivated vaccine: systemic vaccine reactions, local and systemic antibody response, and vaccine efficacy. A meta-analysis. Vaccine $\mathbf{2 0}$ 1340-1353 (2002) 
14. US Department of Health and Human Services Food and Drug Administration Center for Biologics Evaluation and Research. Guidance for Industry: Clinical Data Needed to Support the Licensure of Pandemic Influenza Vaccines (Office of Communication, Training and Manufacturers Assistance, Rockville, Maryland, 2007).

15. Wrammert, J. et al. Rapid cloning of high-affinity human monoclonal antibodies against influenza virus. Nature 453, 667-671 (2008).

16. Takaoka, A. \& Yanai, H. Interferon signalling network in innate defence. Cell. Microbiol. 8, 907-922 (2006).

17. Tusher, V.G., Tibshirani, R. \& Chu, G. Significance analysis of microarrays applied to the ionizing radiation response. Proc. Natl. Acad. Sci. USA 98, 5116-5121 (2001).

18. Iwakoshi, N.N. et al. Plasma cell differentiation and the unfolded protein response intersect at the transcription factor XBP-1. Nat. Immunol. 4, 321-329 (2003).

19. Iwakoshi, N.N., Lee, A.H. \& Glimcher, L.H. The X-box binding protein-1 transcription factor is required for plasma cell differentiation and the unfolded protein response. Immunol. Rev. 194, 29-38 (2003).

20. Ron, D. \& Walter, P. Signal integration in the endoplasmic reticulum unfolded protein response. Nat. Rev. Mol. Cell Biol. 8, 519-529 (2007).

21. Ueda, Y. et al. Frequencies of dendritic cells (myeloid DC and plasmacytoid DC) and their ratio reduced in pregnant women: comparison with umbilical cord blood and normal healthy adults. Hum. Immunol. 64, 1144-1151 (2003).

22. Shen-Orr, S.S. et al. Cell type-specific gene expression differences in complex tissues. Nat. Methods 7, 287-289 (2010).

23. Avery, D.T. et al. BAFF selectively enhances the survival of plasmablasts generated from human memory B cells. J. Clin. Invest. 112, 286-297 (2003).

24. Park, S.W. et al. The regulatory subunits of PI3K, p85alpha and p85beta, interact with XBP-1 and increase its nuclear translocation. Nat. Med. 16, 429-437 (2010).

25. Liu, B. \& Li, Z. Endoplasmic reticulum HSP90b1 (gp96, grp94) optimizes B-cell function via chaperoning integrin and TLR but not immunoglobulin. Blood 112, 1223-1230 (2008).

26. Apostolou, A., Shen, Y., Liang, Y., Luo, J. \& Fang, S. Armet, a UPR-upregulated protein, inhibits cell proliferation and ER stress-induced cell death. Exp. Cell Res. 314, 2454-2467 (2008)

27. Huleatt, J.W. et al. Potent immunogenicity and efficacy of a universal influenza vaccine candidate comprising a recombinant fusion protein linking influenza M2e to the TLR5 ligand flagellin. Vaccine 26, 201-214 (2008).

28. Treanor, J.J. et al. Safety and immunogenicity of a recombinant hemagglutinin influenza-flagellin fusion vaccine (VAX125) in healthy young adults. Vaccine $\mathbf{2 8}$, 8268-8274 (2010).

29. Talbot, H.K. et al. Immunopotentiation of trivalent influenza vaccine when given with VAX102, a recombinant influenza M2e vaccine fused to the TLR5 ligand flagellin. PLOS ONE 5, e14442 (2010).

30. He, X.S. et al. Cellular immune responses in children and adults receiving inactivated or live attenuated influenza vaccines. J. Virol. 80, 11756-11766 (2006).

31. Le Bon, A. et al. Cutting edge: enhancement of antibody responses through direct stimulation of B and T cells by type I IFN. J. Immunol. 176, 2074-2078 (2006).

32. Lee, E.K. Large-scale optimization-based classification models in medicine and biology. Ann. Biomed. Eng. 35, 1095-1109 (2007).
33. Brooks, J.P. \& Lee, E.K. Analysis of the consistency of a mixed integer programming based multi-category constrained discriminant model. Ann. Oper. Res. 174, 147-168 (2010).

34. Sullivan, S.J., Jacobson, R. \& Poland, G.A. Advances in the vaccination of the elderly against influenza: role of a high-dose vaccine. Expert Rev. Vaccines 9 , 1127-1133 (2010).

35. Anderson, K.J. \& Allen, R.L. Regulation of T-cell immunity by leucocyte immunoglobulin-like receptors: innate immune receptors for self on antigenpresenting cells. Immunology 127, 8-17 (2009).

36. Thomas, R., Matthias, T. \& Witte, T. Leukocyte immunoglobulin-like receptors as new players in autoimmunity. Clin. Rev. Allergy Immunol. 38, 159-162 (2010).

37. Brown, D., Trowsdale, J. \& Allen, R. The LILR family: modulators of innate and adaptive immune pathways in health and disease. Tissue Antigens 64, 215-225 (2004).

38. Krebs, J., Wilson, A. \& Kisielow, P. Calmodulin-dependent protein kinase IV during T-cell development. Biochem. Biophys. Res. Commun. 241, 383-389 (1997).

39. Wang, S.L., Ribar, T.J. \& Means, A.R. Expression of $\mathrm{Ca}^{2+} /$ calmodulin-dependent protein kinase IV (caMKIV) messenger RNA during murine embryogenesis. Cell Growth Differ. 12, 351-361 (2001).

40. Anderson, K.A. \& Means, A.R. Defective signaling in a subpopulation of CD4 $\mathrm{T}$ cells in the absence of $\mathrm{Ca}^{2+} /$ calmodulin-dependent protein kinase IV. Mol. Cell. Biol. 22, 23-29 (2002).

41. Illario, M. et al. Calmodulin-dependent kinase IV links Toll-like receptor 4 signaling with survival pathway of activated dendritic cells. Blood 111, 723-731 (2008).

42. Sato, K. et al. Regulation of osteoclast differentiation and function by the CaMKCREB pathway. Nat. Med. 12, 1410-1416 (2006).

43. Kitsos, C.M. et al. Calmodulin-dependent protein kinase IV regulates hematopoietic stem cell maintenance. J. Biol. Chem. 280, 33101-33108 (2005).

44. Pulendran, B. \& Ahmed, R. Immunological mechanisms of vaccination. Nat. Immunol. 131, 509-517 (2011).

45. Moldoveanu, Z., Clements, M.L., Prince, S.J., Murphy, B.R. \& Mestecky, J. Human immune responses to influenza virus vaccines administered by systemic or mucosal routes. Vaccine 13, 1006-1012 (1995).

46. Shubinsky, G. \& Schlesinger, M. The CD38 lymphocyte differentiation marker: new insight into its ectoenzymatic activity and its role as a signal transducer. Immunity 7 , 315-324 (1997).

47. Deaglio, S., Mehta, K. \& Malavasi, F. Human CD38: a (r)evolutionary story of enzymes and receptors. Leuk. Res. 25, 1-12 (2001).

48. Clements, M.L., Betts, R.F., Tierney, E.L. \& Murphy, B.R. Serum and nasal wash antibodies associated with resistance to experimental challenge with influenza $A$ wild-type virus. J. Clin. Microbiol. 24, 157-160 (1986).

49. Potter, C.W. \& Oxford, J.S. Determinants of immunity to influenza infection in man. Br. Med. Bull. 35, 69-75 (1979).

50. Hirota, Y. et al. Antibody efficacy as a keen index to evaluate influenza vaccine effectiveness. Vaccine 15, 962-967 (1997).

51. Belshe, R.B. Current status of live attenuated influenza virus vaccine in the US Virus Res. 103, 177-185 (2004). 


\section{ONLINE METHODS}

Clinical study. This study included subjects vaccinated with TIV during the three consecutive influenza seasons in 2008-2009 (trial $1 ; n=28$ ), 2007-2008 (trial 2; $n=9$ ) and 2009-2010 (trial 3; $n=30$ ); one trial included subjects vaccinated with LAIV during 2008-2009 influenza season. Young healthy adults 18-50 years old were vaccinated with one dose of TIV (Fluarix (GlaxoSmithKline Biologicals) in 2007-2008 and 2008-2009 or Fluvirin (Novartis Vaccines and Diagnostics) in 2009-2010) or LAIV (FluMist; MedImmune) according to guidelines for influenza vaccination (influenza virus strains in the vaccines, Supplementary Table 10). Written informed consent was obtained from each subject with institutional review and approval from the Emory University Institutional Review Board.

Cells, plasma and RNA isolation. PBMCs and plasma were isolated from fresh blood (in Vacutainer cell preparation tubes with sodium citrate) according to the manufacturer's protocol (BD). Total RNA from fresh PBMCs $\left(\sim 1.5 \times 10^{6}\right.$ cells) was purified with TRIzol according to the manufacturer's instructions (Invitrogen, Life Technologies). The purity of all RNA samples was checked with an ND-1000 spectrophotometer (NanoDrop Technologies) and their integrity was checked by electrophoresis on a 2100 BioAnalyzer (Agilent Technologies).

RT-PCR. Total RNA was reverse-transcribed with a High-Capacity cDNA Archive Kit Protocol (Applied Biosystems). Custom-designed Low Density Array and Quantitative Real-Time PCR Analysis Low Density Arrays for 48 genes were from Applied Biosystems (Supplementary Table 7).

Microarray experiments. Total RNA was hybridized on Human U133 Plus 2.0 arrays (with a GeneTitan platform, Affymetrix or individual cartridges). The intensity microarray data for probe sets (called 'genes' here) was normalized by the robust multi-array average method, which includes global background adjustment and quantile normalization. For microarray analysis of flow cytometry-sorted subsets of immune-response cells, total RNA was amplified with the WT-Ovation Pico RNA Amplification System according to the manufacturer's instructions (NuGEN). Next, cDNA was labeled with an FL-Ovation cDNA Biotin Module V2 kit according to the manufacturer's protocol (NuGEN). The Affymetrix GeneChip Array Station automation platform was used for hybridization to Affymetrix HT Human Genome U133A arrays (Affymetrix) and washing. Arrays were scanned on a GeneChip HT Array Plate Scanner (00-0332; Affymetrix) and data were processed with normalization by robust multi-array averaging. Additional details are in the Supplementary Methods.

HAI assays. HAI titers were determined based on the standard World Health Organization protocol as described ${ }^{52}$. Plasma samples were treated with receptor-destroying enzyme (Denka Seiken) at a ratio of one part plasma to three parts enzyme and incubation overnight at $37^{\circ} \mathrm{C}$. The next morning, the enzyme was inactivated by incubation of the samples for $1 \mathrm{~h}$ at $56^{\circ} \mathrm{C}$. Samples were then serially diluted with PBS in 96-well V-bottomed plates (Nunc), and 4 hemagglutinating units of influenza strain $\mathrm{H} 1 \mathrm{~N} 1$ or $\mathrm{H} 3 \mathrm{~N} 2$ or influenza $\mathrm{B}$ virus was added to each well. After $30 \mathrm{~min}$ at $25^{\circ} \mathrm{C}, 50 \mu \mathrm{l}$ of $0.5 \%$ turkey RBCs (Rockland Immunochemicals) suspended in PBS with $0.5 \%$ (wt/vol) BSA was added to each well and plates were shaken manually. After an additional $30 \mathrm{~min}$ at $25^{\circ} \mathrm{C}$, plasma titers were measured as the reciprocal of the final dilution for which a pellet was observed. Negative and positive control plasmas for each virus were used for reference.

B cell ELISPOT and flow cytometry of B cells. Direct ELISPOT assays for counting both total plasmablasts and plasmablasts expressing influenzaspecific IgG, IgM or IgA in fresh PBMC samples were done essentially as described $^{15,53}$. First, 96-well ELISPOT filter plates (MAHA N4510; Millipore) were coated overnight with vaccine against influenza (same vaccine as that used for donor vaccination) at a dilution of 1:20 in PBS or with goat antibody to human immunoglobulin (H17000; Caltag). Plates were washed, and unoccupied sites on the filters were blocked by incubation for $2 \mathrm{~h}$ at $37^{\circ} \mathrm{C}$ with complete RPMI medium containing $10 \%$ (vol/vol) FCS. Purified and extensively washed PBMCs were added to the plates in serial dilutions, followed by incubation for $6 \mathrm{~h}$ or overnight. Plates were washed with PBS followed by PBS containing $0.05 \%$ (vol/vol) Tween and then were incubated for $1.5 \mathrm{~h}$ at $25^{\circ} \mathrm{C}$ with biotinylated antibody to human IgG (H10015; Invitrogen), IgA (H14015; Invitrogen) or IgM (H15015; Invitrogen). After being washed, plates were incubated with an avidin D-horseradish peroxidase conjugate (A-2004; Vector Laboratories) and were finally developed with AEC substrate (3 amino-9 ethylcarbozole; Sigma). Developed plates were scanned and analyzed with an automated ELISPOT counter (Cellular Technologies). Flow cytometry analysis was done on whole blood as described ${ }^{15}$. Before analysis, red blood cells were lysed twice ( 4 min each) at $25^{\circ} \mathrm{C}$ by incubation with BD FACS Lysing Solution (Becton Dickinson). Blood (300-400 $\mu \mathrm{l}$ ) was then incubated for $30 \mathrm{~min}$ at $25^{\circ} \mathrm{C}$ with the appropriate antibodies: antibody to CD3 (anti-CD3; 557851; Pharmingen), anti-CD20 (335793; Pharmingen), anti-CD38 (555460; Pharmingen), anti-CD19 (555412; Pharmingen) and anti-CD27 (17-0279; eBioscience). ASCs were gated and isolated as $\mathrm{CD} 19^{+} \mathrm{CD} 3^{-} \mathrm{CD} 20^{\text {lo-neg }} \mathrm{CD} 27^{\text {hi }}$ $\mathrm{CD} 38^{\text {hi }}$ cells. Flow cytometry data were analyzed with FlowJo software.

Meta-analysis of immune-response cells and microarray analysis. Full details of these procedures (including sorting of subsets of immune-response cell by flow cytometry and identification of genes with differences in expression in PBMCs and flow cytometry-sorted cell subsets; meta-analysis; correlation and predictive analyses) are in the Supplementary Methods.

Analysis of immune responses in $\mathrm{Camk4}^{-/-}$mice. C57BL/6 mice 8-12 weeks old were from The Jackson Laboratory. Camk $4^{-1-}$ mice were generated as described ${ }^{54}$ and were back-bred to C57BL/6J mice for more than 12 generations. Mice were immunized in the right and left hamstring muscles with the human Fluvirin vaccine (Novartis Vaccines and Diagnostics) diluted 1:5. Blood was obtained from mice at various time points for analysis of influenza-specific antibody responses. All animal procedures were done in accordance with guidelines established by the Institutional Animal Care and Use Committee of Duke University.

For enzyme-linked immunosorbent assay, 96-well Maxisorp plates (Nunc) were coated overnight at $4{ }^{\circ} \mathrm{C}$ with Fluvirin (for trials in 2009-2010 and 2010$2011 ; 2 \mu \mathrm{g} / \mathrm{ml}$ in a volume of $100 \mu \mathrm{l}$; Novartis Vaccines and Diagnostics). Plates were washed three times with $5 \%$ (vol/vol) Tween 20 in PBS with a Biotek Auto Plate Washer, followed by incubation for $2 \mathrm{~h}$ at $25^{\circ} \mathrm{C}$ with $200 \mu \mathrm{l}$ per well of a solution of $4 \%(\mathrm{wt} / \mathrm{vol})$ nonfat dry milk (Bio-Rad). Serum samples obtained from immunized mice at various time points were diluted (1:100) in $0.1 \%$ (wt/ vol) nonfat dry milk in a solution of $0.5 \%$ (vol/vol) Tween 20 in PBS and were incubated for $2 \mathrm{~h}$ at $25^{\circ} \mathrm{C}$ on blocked plates. Plates were washed five times after incubation of sample serum, and horseradish peroxidase-conjugated antibody to mouse IgG2c (1:2,000 dilution; 1079-05; SouthernBiotech) and to mouse IgG1 (1:5,000 dilution; 1070-05; SouthernBiotech) in 0.5\% (vol/vol) Tween-20 in PBS were added, then plates were incubated for $2 \mathrm{~h}$ at $25^{\circ} \mathrm{C}$. Plates were washed seven times with $0.5 \%$ (vol/vol) Tween-20 in PBS and were developed with tetramethylbenzidine substrate ( $100 \mu \mathrm{l}$ per well; BD Biosciences), then reactions were stopped with $2 \mathrm{~N} \mathrm{H}_{2} \mathrm{SO}_{4}$. Plates were analyzed with a BioRad plate reading spectrophotometer at $450 \mathrm{~nm}$ with correction at $595 \mathrm{~nm}$. Results are presented as absorbance at $450 \mathrm{~nm}$. Details of immunoblot analyses are in the Supplementary Methods.

52. Chen, G.L., Lamirande, E.W., Jin, H., Kemble, G. \& Subbarao, K. Safety, immunogencity, and efficacy of a cold-adapted A/Ann Arbor/6/60 (H2N2) vaccine in mice and ferrets. Virology 398, 109-114 (2010).

53. Crotty, S. et al. Cutting edge: long-term B cell memory in humans after smallpox vaccination. J. Immunol. 171, 4969-4973 (2003).

54. Wu, J.Y. et al. Spermiogenesis and exchange of basic nuclear proteins are impaired in male germ cells lacking Camk4. Nat. Genet. 25, 448-452 (2000). 